\title{
Zinc transporter 8 (ZnT8) autoantibody prevalence in black South African participants with type 1 diabetes
}

\author{
Sureka Bhola ${ }^{1,2^{*}}$, Eleanor M Cave ${ }^{1}$, Sindeep Bhana ${ }^{3}$, Nigel J Crowther ${ }^{1,2}$ and Carolyn J Padoa ${ }^{1,2}$
}

\begin{abstract}
Background: Autoantibodies to $\beta$-cell specific antigens are markers of type 1 diabetes. The most recently identified autoantibodies are targeted to the zinc transporter 8 (ZnT8) protein located in the membrane of $\beta$-cell insulin secretory granules. The prevalence of ZnT8 autoantibodies in newly diagnosed participants with type 1 diabetes has been found to range from 33 to $80 \%$. Due to the lack of data on the immunological aetiology of type 1 diabetes in African populations, this study aimed to determine the prevalence of ZnT8 autoantibodies in black South Africans with type 1 diabetes and whether ZnT8 autoantibody positivity was associated with age at diagnosis and disease duration.
\end{abstract}

Methods: Participants with type 1 diabetes and controls were recruited from the greater Johannesburg area, South Africa. Positivity for ZnT8, GAD65 and IA2 autoantibodies was determined by ELISA.

Results: Participants with type 1 diabetes $(n=183)$ and controls $(n=49)$ were matched for age $(29.1 \pm 9.53$ vs. $27.3 \pm 7.29$, respectively; $p=0.248$ ). The mean age at diagnosis for participants with type 1 diabetes was $20.8 \pm 8.46$ years. The prevalence of ZnT8 autoantibody positivity was $17.5 \%$ (32 of 183) in participants with type 1 diabetes with a median disease duration of 7.00 [2.00; 11.0] years. ZnT8 autoantibody prevalence in newly diagnosed participants (< 1 year duration) was $27.3 \%$ (6 of 22). Logistic regression analysis found an association between ZnT8 autoantibody positivity and shorter disease duration (OR: 0.9 (0.81-1.00); $\mathrm{p}=0.042$ ). In addition, ZnT8 autoantibody positivity was significantly associated with an increased chance of being GAD65 (OR: 3.37 (1.10-10.3)) and IA2 (OR: 8.63 (2.82-26.4)) autoantibody positive. Multiple regression analysis found no association between ZnT8 autoantibody positivity and age at diagnosis. However, the presence of $\geq 2$ autoantibodies was associated with a younger age at diagnosis of type 1 diabetes when compared to participants with $\leq 1$ autoantibody $(B=-5.270 ; p=$ 0.002).

Conclusions: The presence of ZnT8 autoantibodies was not related to a younger age at diagnosis in black South African patients with type 1 diabetes. However, the greater the numbers of autoantibodies present in an individual the earlier the age at diagnosis. ZnT8 autoantibodies decline with disease duration in the black South African population.

Keywords: ZnT8, Autoantibody, Type 1 diabetes, Black South African, Prevalence

\footnotetext{
*Correspondence: sureka.bhola@nhls.ac.za

'Department of Chemical Pathology, Faculty of Health Sciences, University of

the Witwatersrand, Johannesburg, South Africa

${ }^{2}$ National Health Laboratory Service, Johannesburg, South Africa

Full list of author information is available at the end of the article
}

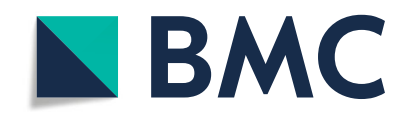

(c) The Author(s). 2021 Open Access This article is licensed under a Creative Commons Attribution 4.0 International License, which permits use, sharing, adaptation, distribution and reproduction in any medium or format, as long as you give appropriate credit to the original author(s) and the source, provide a link to the Creative Commons licence, and indicate if changes were made. The images or other third party material in this article are included in the article's Creative Commons licence, unless indicated otherwise in a credit line to the material. If material is not included in the article's Creative Commons licence and your intended use is not permitted by statutory regulation or exceeds the permitted use, you will need to obtain permission directly from the copyright holder. To view a copy of this licence, visit http://creativecommons.org/licenses/by/4.0/ The Creative Commons Public Domain Dedication waiver (http://creativecommons.org/publicdomain/zero/1.0/) applies to the data made available in this article, unless otherwise stated in a credit line to the data. 


\section{Background}

Type 1 diabetes (T1D) is a chronic autoimmune disease which results in the destruction of the insulin secreting $\beta$-cells in the pancreatic islets of Langerhans [1]. The disease is characterised by the presence of autoantibodies (AAbs) to beta-cell specific antigens which may develop years before the clinical diagnosis of the disease $[2,3]$. Autoantibodies directed to the zinc transporter 8 (ZnT8) peptide have been described in T1D [4]. This peptide is encoded by the SLC308 gene located on chromosome 8q24.11 and is found in the membrane of insulin secretory granules located in pancreatic $\beta$-cells [5]. The $\mathrm{ZnT} 8$ peptide mediates the uptake of $\mathrm{Zn}^{2+}$ into the secretory granules which in turn stabilises insulin by allowing hexamer formation $[6,7]$.

Several studies have determined the prevalence of ZnT8 AAb positivity in T1D, which varies with ethnicity. In Europeans, the prevalence of ZnT8 AAbs ranged between 60 and $80 \%$ of newly diagnosed participants with T1D [4, 8-11]. In comparison, Asian populations reported relatively low ZnT8 AAb prevalences in newly diagnosed individuals with T1D, with a Chinese and a Japanese study reporting prevalences of $32.9 \%$ (48 of 146 ) and $36 \%$ (40 of 112), respectively [12, 13]. The T1D Genetics Consortium found that in newly diagnosed non-Hispanic black participants (duration $<3$ years) the ZnT8 prevalence was $57.9 \%$ (Wenzlau et al., 2015).

The prevalence of ZnT8 AAbs has been shown to decline with increasing disease duration. A study by Howson et al., [14] conducted in British participants with T1D showed that ZnT8 AAb positivity decreased from $58 \%$ in participants with a disease duration $\leq 2$ years to $10 \%$ in those with a disease duration $\geq 9$ years. Fabris and colleagues found that in an Italian study, the prevalence of ZnT8 AAbs remained stable for the first 4 years after diagnosis of T1D (61.1\% at onset, $56.0 \%$ at 1 year and $59.3 \%$ at $2-4$ years) and then dropped significantly to $33.8 \%$ at $\geq 5$ years [10]. Similar declines in ZnT8 AAb frequency with increasing disease duration were seen in studies conducted in Brazilian, Japanese and nonHispanic white populations $[9,15,16]$.

The ZnT8 AAbs are more prevalent in newly diagnosed children than adults with T1D. Thus, a Swedish study found the prevalence of $\mathrm{ZnT} 8 \mathrm{AAb}$ positivity to be $66 \%$ (227 of 343) in newly diagnosed participants with an age at onset between 15 and 34 years compared to $80 \%$ (200 of 249) in a younger cohort with an age at onset of 2-17 years [8]. In a Polish cohort of newly diagnosed individuals with T1D, ZnT8 AAbs were more prevalent in children (81.1\% [177 of 218], median age 9 years [interquartile range: $6-13$ years]) than in adults (34.8\% [52 of 149], median age 34 years [interquartile range: 27-43 years]) [17]. Similar associations of ZnT8
AAb positivity with younger age at diagnosis have been observed in Asian populations [12, 16].

Few studies have assessed the frequency of ZnT8 AAb positivity in sub-Saharan African populations. In a cohort of 85 newly diagnosed Ugandan individuals with T1D ( $<25$ years old), the prevalence of ZnT8 AAb positivity was $17.6 \%$ [18]. A study conducted on newly diagnosed Ethiopians with T1D ( $\leq 35$ years) found an overall frequency of $10.2 \%$ (24 of 236) for ZnT8 AAbs. Although there was a decline in ZnT8 AAb positivity with an increasing age at diagnosis it did not reach significance ( $0-15$ years: $16.7 \%$ (6 of 36 ) vs. $16-35$ years: $9.0 \%$ (18 of 200, $p=0.161$ ) [19]. A study conducted in Somalian migrants living in America found a ZnT8 AAb prevalence of $26 \%$ (8 of 31) in participants with T1D with an age at onset $\leq 19$ years [20].

Due to the lack of studies on ZnT8 AAbs in T1D in sub-Saharan Africa, this study aimed to determine the prevalence of ZnT8 AAbs in black South Africans with T1D. In addition, we aimed to determine whether ZnT8 AAb positivity was associated with age at diagnosis and disease duration.

\section{Methods}

\section{Participant recruitment}

Clinically diagnosed black South African participants with T1D (cases; $\mathrm{n}=183$ ) were recruited from diabetes clinics at Chris Hani Baragwanath Academic Hospital and Charlotte Maxeke Johannesburg Academic Hospital between November 2014 and December 2015. The study protocol was approved by the University of the Witwatersrand Human Research Ethics Committee (clearance certificate numbers M180334 and M150885) and by the South African Blood Transfusion Services Research Ethics Committee (clearance certificate number 2014/19). The study protocol conformed to the Declaration of Helsinki ethical guidelines. Informed written consent was obtained from all participants prior to commencement of the study. Black participants that were not diabetic (controls; $\mathrm{n}=49$ ) were recruited from the South African Blood Transfusion Services blood drives and from students and staff based at the University of Witwatersrand Medical School in Johannesburg. Participants were classified as controls if they had a random plasma blood glucose level $<11.1 \mathrm{mmol} / \mathrm{L}$ and were not on any glucose lowering medication. Anthropometric measurements were taken for all participants. Patient glycated haemoglobin (HbA1c) and glucose levels were obtained from patient files. Participants with clinical evidence of chronic pancreatitis, gestational diabetes or type 2 diabetes were excluded from the study. 


\section{Measurement of glucose concentrations}

Random plasma glucose concentrations for control participants were measured on the ADVIA Chemistry System (Siemens Health Care Diagnostics Inc., New York, USA) in the National Health Laboratory Services Chemical Pathology Diagnostic Laboratory based at Charlotte Maxeke Johannesburg Academic Hospital using the enzymatic hexokinase method.

\section{Measurement of ZnT8, GAD65 and IA2 AAbs}

The $\mathrm{ZnT} 8,65 \mathrm{kDa}$ isoform of glutamic acid decarboxylase (GAD65) and protein tyrosine phosphatase related islet antigen 2 (IA2) AAb status was determined using ELISA kits (KRONUS, Idaho, USA) according to the manufacturer's instructions. Based on the manufacturer's guidelines, participants with ZnT8 and IA2 AAb concentrations $\geq 15 \mathrm{U} / \mathrm{ml}$, and GAD65 AAb concentrations $\geq 5$ $\mathrm{IU} / \mathrm{ml}$ were classified as positive.

\section{Statistical analysis}

Continuous data that were not normally distributed were $\log$ transformed to normality with the exception of duration of disease for which the square-root was used. Normally distributed continuous variables were presented as mean \pm standard deviation (SD) whilst skewed data were presented as median [lower quartile; upper quartile]. Categorical variables were presented as percentages (\%). The two-tailed Student's non-paired t-test was used to compare variables between participants with T1D who were AAb positive and those who were $A A b$ negative. A chi squared $\left(x^{2}\right)$ test was used to compare all categorical variables. Backwards, stepwise multivariable logistic regression analyses were performed using $\mathrm{ZnT}$ 8 $\mathrm{AAb}$ positivity as the dependent variable and independent variables chosen based on scientific plausibility and their association $(p<0.20)$ with AAb positivity in univariate analyses. Backward stepwise multivariable linear regression analysis was similarly performed with age at diagnosis as the dependent variable. Results with a $p$ value $<0.05$ were considered to be statistically significant. All statistical analyses were performed using Statistica software version 13 (StatSoft, Tulsa, Oklahoma, USA).

\section{Results}

\section{Clinical and phenotypic characteristics of patients with T1D and control participants}

The clinical and phenotypic characteristics of the study participants are summarised in Table 1 . The patients with T1D had significantly more males ( 54.6 vs. $34.7 \%$; $p=0.013$ ) and had higher glucose levels (9.20 [5.70; $13.2]$ vs. $5.00[4.50 ; 6.00] \mathrm{mmol} / \mathrm{L} ; p<0.001)$ and a higher frequency of $\mathrm{ZnT} 8$ (17.5 vs. $2.0 \% ; p=0.006)$ and GAD65 AAb positivity (51.3 vs. $2.1 \%$; $p<0.001)$ when
Table 1 Clinical and phenotypic characteristics of the study participants

\begin{tabular}{llll}
\hline Variables & $\begin{array}{l}\text { Cases } \\
(\mathbf{n}=\mathbf{1 8 3})\end{array}$ & $\begin{array}{l}\text { Controls } \\
(\mathbf{n}=\mathbf{4 9})\end{array}$ & $\boldsymbol{p}$ value \\
\hline Age (years) & $29.1 \pm 9.53$ & $27.3 \pm 7.29$ & 0.248 \\
Age at diagnosis (year) & $20.8 \pm 8.46$ & - & - \\
Duration of disease (years) & $7.00[2.00 ; 11.0]$ & - & - \\
Gender & & & 0.013 \\
males, \% (n) & $54.6(100)$ & $34.7(17)$ & \\
Females, \% (n) & $45.4(83)$ & $65.3(32)$ & - \\
Glucose (mmol/L) & $9.20[5.70 ; 13.2]^{a}$ & $5.00[4.50 ; 6.00]$ & $<0.001$ \\
HbA1c (\%) & $10.6 \pm 3.41$ & - & 0.023 \\
BMI (kg/m ${ }^{2}$ ) & $23.7[21.2 ; 27.8]$ & $25.4[23.4 ; 28.2]$ & 0.006 \\
ZnT8 AAb positivity (\%; n) & $17.5(32)$ & $2.0(1)$ & $<0.001$ \\
GAD65 AAb positivity (\%; n) & $51.3(80)^{b}$ & $2.1(1)^{c}$ & 0.237 \\
\hline IA2 AAb positivity (\%; $n)$ & $12.8(20)^{b}$ & $6.5(3)^{d}$ & \\
\hline
\end{tabular}

Results are presented as median values [lower quartile; upper quartile] for skewed data and as mean \pm standard deviation for non-skewed data and $\%$ (n) for categorical variables; Missing data: ${ }^{a} n=14,{ }^{b} n=27,{ }^{c} n=2,{ }^{d} n=3$

compared to controls. The BMI was significantly higher in the control group than in the patient group (25.4 [23.4; 28.2] vs. 23.7 [21.2; 27.8]; $p=0.023$ ). The mean age at diagnosis of T1D was $20.8 \pm 8.46$ years with a median duration of disease of $7.00[2.00 ; 11.0]$ years.

\section{Autoantibody positivity, age at diagnosis and disease duration}

The association of clinical characteristics in the participants with T1D with ZnT8 AAb positivity can be seen in Table 2. The ZnT8 AAb positivity was associated with a younger age at diagnosis $(17.0 \pm 6.49$ vs. $21.6 \pm 8.62$ years, $p=0.005)$, a shorter duration of disease $(3.50$ [1.00; 8.00] vs. $7.00[3.00 ; 12.0]$ years; $p=0.003)$, higher glucose concentrations $(10.9[7.10 ; 16.1]$ vs. $8.40[5.40$; 13.1] $\mathrm{mmol} / \mathrm{L} ; p=0.042)$ and a lower BMI (20.1 [19.4; $23.9]$ vs. 23.9 [21.4; 28.7$\left.] \mathrm{kg} / \mathrm{m}^{2} ; p=0.003\right)$. In addition, ZnT8 AAb positive participants had significantly higher IA2 (44.4 vs. $6.2 \%$; < 0.001 ) and GAD65 (81.5 vs. 45.0 $\%$; $p<0.001)$ AAb positivity when compared to ZnT8 AAb negative participants.

There was no significant difference in ZnT8 and IA2 $\mathrm{AAb}$ frequencies within the first three years following T1D diagnosis $(p=0.961, \mathrm{p}=0.729$, respectively), whereas significant differences were observed for GAD65 AAb positivity $(p=0.049)$. However, there was a significant decline in ZnT8 and GAD65 AAb frequencies between participants with a disease duration $\leq 3$ years and those with a disease duration $\geq 4$ years (25.4 vs. 13.4 $\% ; p=0.044$ and 66.7 vs. $44.2, p=0.009$, respectively) (Table 3). However, the median ZnT8 AAb titres in participants who tested positive for this AAb and with disease duration $\leq 3$ years $(173.4[42.7 ; 227.2] \mathrm{U} / \mathrm{ml})$ was 
Table 2 Association of ZnT8 AAb positivity with clinical and phenotypic variables in participants with T1D

\begin{tabular}{|c|c|c|c|}
\hline \multirow[t]{2}{*}{ Variable } & \multicolumn{2}{|c|}{ ZnT8 AAb positivity } & \multirow[t]{2}{*}{$p$ value } \\
\hline & Positive $(n=32)$ & Negative $(n=151)$ & \\
\hline Age at diagnosis (years) & $17.0 \pm 6.49$ & $21.6 \pm 8.62$ & 0.005 \\
\hline Duration of disease (years) & $3.50[1.00 ; 8.00]$ & $7.00[3.00 ; 12.0]$ & 0.003 \\
\hline \multicolumn{4}{|l|}{ Gender } \\
\hline Males, \% (n) & $59.4(19)$ & $53.6(81)$ & \multirow[t]{2}{*}{0.696} \\
\hline Females, \% (n) & $40.6(13)$ & $46.4(70)$ & \\
\hline Glucose (mmol/L) & $10.9[7.10 ; 16.1]^{\mathrm{a}}$ & $8.40[5.40 ; 13.1]^{b}$ & 0.042 \\
\hline $\mathrm{HbA1c}(\%)$ & $10.9 \pm 3.50$ & $10.5 \pm 3.40$ & 0.593 \\
\hline $\mathrm{BMI}\left(\mathrm{kg} / \mathrm{m}^{2}\right)$ & $20.1[19.4 ; 23.9]$ & $23.9[21.4 ; 28.7]$ & 0.003 \\
\hline IA2 AAb positivity (\%; n) & $44.4(12)^{c}$ & $6.2(8)^{d}$ & $<0.001$ \\
\hline GAD65 AAb positivity (\%; n) & $81.5(22)^{c}$ & $45.0(58)^{d}$ & $<0.001$ \\
\hline
\end{tabular}

Results are presented as median values [interquartile range] for skewed data and as mean \pm standard deviation for non-skewed data; Missing data: ${ }^{a} n=4,{ }^{b} n=$ $10, c^{c}=5$ and ${ }^{d} n=22$

not significantly different from those with disease duration $\geq 4$ years $(125.2$ [76.2; 403.7] $\mathrm{U} / \mathrm{ml} ; p=0.580)$.

The greater the number of AAbs present in an individual, the younger the age at diagnosis $(p=0.008)$ and the shorter the disease duration $(p=0.022)$ (Table 4$)$. In addition, the presence of AAbs (irrespective of number) was associated with a lower BMI $(p=0.040)$.

In order to identify the main determinants of ZnT8 AAb positivity, relevant variables that associated with the presence of these AAbs at $p<0.20$ (i.e. disease duration, BMI, GAD65 and IA2 AAb positivity; see Table 2) were included in a backward stepwise multivariate logistic regression analysis (Table 5). This showed that ZnT8 AAb positivity was associated with a shorter disease duration (OR (95\% CIs): 0.90 (0.81-1.00); $p=0.042)$. In addition, both GAD65 and IA2 $\mathrm{AAb}$ positivity were associated with ZnT8 AAb positivity ( $p=0.033$ and $p<0.001$, respectively).

The possible determinants of age at diagnosis were identified using a backward stepwise multivariable linear regression analysis using relevant independent variables that correlated with age at diagnosis in a Pearson correlation at $p<0.20$ (i.e. gender, ZnT8, GAD65 and IA2 AAb positivity; Table 6). In this model ZNT8 AAb positivity was not significantly associated with age at diagnosis, with only IA2 AAb positivity showing a significant negative association $(p=0.005)$ and gender tending toward significance $(p=0.052)$

To determine whether the number of AAbs present affected age at diagnosis, backward stepwise multivariable linear regression analysis controlling for gender was performed (Table 7). The presence of two or more AAbs was associated with an age at diagnosis five years younger than participants with zero or one AAb $(p=0.002)$. This model accounted for $6.7 \%$ of the factors contributing to age at diagnosis.

\section{Discussion}

There is a paucity of data regarding the prevalence of T1D-associated AAbs in sub-Saharan African populations. In the current study, the prevalence of ZnT8 AAbs in black South Africans with T1D (median disease duration of seven years) was $17.5 \%$ whereas in the control population the prevalence was only $2 \%$. In newly diagnosed participants $(<1$ year duration) the prevalence was $27.3 \%$ ). When comparing participants with a disease duration $\leq 3$ years to those with a longer disease duration, ZnT8 and GAD65 AAb frequency declined ( $p=$ 0.044 and 0.009 , respectively). The association of ZnT8 AAb positivity and disease duration was confirmed in a logistic regression model $(p=0.042)$. Participants who

Table 3 Autoantibody frequency in patients with T1D according to duration of disease

\begin{tabular}{|c|c|c|c|c|c|c|c|}
\hline & \multicolumn{7}{|c|}{ Duration of disease } \\
\hline & $\begin{array}{l}<1 \text { year } \\
(n=22)\end{array}$ & $\begin{array}{l}1-2 \text { years } \\
(n=28)\end{array}$ & $\begin{array}{l}3 \text { years } \\
(n=13)\end{array}$ & $p$ value & $\begin{array}{l}\leq 3 \text { years } \\
(n=63)\end{array}$ & $\begin{array}{l}\geq 4 \text { years } \\
(n=119)\end{array}$ & $p$ value \\
\hline ZnT8 AAb positivity $(\%, n)$ & $27.3(6)$ & $25.0(7)$ & $23.1(3)$ & 0.961 & $25.4(16)$ & $13.4(16)$ & 0.044 \\
\hline GAD65 AAb positivity $(\%, n)$ & $50.0(9)^{\mathrm{a}}$ & $85.7(18)^{b}$ & $58.3(7)^{c}$ & 0.049 & $66.7(34)^{d}$ & $44.2(46)^{\mathrm{e}}$ & 0.009 \\
\hline IA2 AAb positivity $(\%, n)$ & $16.7(3)^{\mathrm{a}}$ & $19.0(4)^{b}$ & $0.00(0)^{c}$ & 0.729 & $13.7(7)^{d}$ & $12.5(13)^{\mathrm{e}}$ & 0.831 \\
\hline
\end{tabular}

Missing data: ${ }^{a} n=3,{ }^{b} n=7,{ }^{c} n=1,{ }^{d} n=12,{ }^{e} n=15$ 
Table 4 Associations of number of autoantibodies and clinical characteristics in patients with T1D

\begin{tabular}{lllll}
\hline Variable & 0 AAbs $(\mathbf{n}=\mathbf{6 9})$ & 1AAb $(\mathbf{n}=\mathbf{5 7})$ & 2 or $\mathbf{3}$ AAbs $(\mathbf{n}=\mathbf{3 0})$ & $\boldsymbol{p}$ value \\
\hline Age at diagnosis (years) & $22.6 \pm 8.00^{\mathrm{a}}$ & $21.4 \pm 9.62^{\mathrm{a}}$ & $16.9 \pm 6.02$ & 0.008 \\
Duration of disease (years) & $8.00[4.00 ; 13.0]^{\mathrm{a}}$ & $5.00[2.00 ; 12.0]$ & $4.00[1.00 ; 8.00]$ & 0.022 \\
BMI $\left(\mathrm{kg} / \mathrm{m}^{2}\right)$ & $25.2[21.8 ; 29.9]^{\mathrm{a}}$ & $22.5[20.3 ; 25.3]^{\mathrm{a}}$ & $22.9[21.6 ; 25.7]^{\mathrm{a}}$ & 0.040 \\
Glucose $(\mathrm{mmol} / \mathrm{L})$ & $8.25[5.15 ; 13.0]^{\mathrm{b}}$ & $8.10[5.40 ; 13.1]^{\mathrm{b}}$ & $10.9[5.80 ; 17.1]^{\mathrm{c}}$ & 0.249 \\
HbA1c (\%) & $9.87 \pm 3.14^{\mathrm{d}}$ & $10.8 \pm 3.32^{\mathrm{c}}$ & $10.6 \pm 3.46^{\mathrm{e}}$ & 0.255 \\
\hline
\end{tabular}

Results are presented as median values [interquartile range] for skewed data and as mean \pm standard deviation for non-skewed data; Missing data: ${ }^{a} \mathrm{n}=1,{ }^{b} \mathrm{n}=5$, ${ }^{c} n=4,{ }^{d} n=6,{ }^{e} n=2$

were GAD65 and IA2 positive had a 3.37 and an 8.63 times increased likelihood of being ZnT8 positive, respectively. In participants with T1D, ZnT8 AAb positivity was associated with a younger age at diagnosis, however, this association was lost in regression analysis whereas IA2 AAb positivity remained associated with a younger age at diagnosis $(p=0.005)$. In addition, a younger age at diagnosis was found in participants with $\geq 2$ AAbs compared to those with $\leq 1$ AAbs. To our knowledge, this is the first report of $\mathrm{ZnT} 8$ AAbs in black South Africans with T1D.

The overall prevalence of ZnT8 AAb positivity (17.5\%; median duration 7 years) in our cohort was lower but not significantly $(p>0.05)$ different to that in a Chinese population (24.1\% (130 of 539), median disease duration of 2 (range 0-348) months and Somalian immigrants living in America (25.8\% (8 of 31), median disease duration of 3.1 years) $[12,20]$. However, our population had significantly $(p<0.05)$ lower frequencies of $\mathrm{ZnT}$ AAb than Japanese ( $28 \%$ (75 of 270), median disease duration of 2 years), and British (33.6\% (752 of 2237), median disease duration of 4 years) populations $[13,14]$. In our cohort, the prevalence of ZnT8 AAbs in newly diagnosed ( $<1$ year) black South Africans with T1D (27.3\%, 6 of 22) is higher but not significantly $(p>0.05)$ different to that reported in a Ugandan cohort newly diagnosed with T1D (17.6\% (15 of 85)) [18], but is significantly $(p<0.05)$ higher than ZnT8 AAb frequencies reported in newly diagnosed Ethiopians with T1D (10.2\% (24 of 236), $p=0.017)$, and significantly lower than nonHispanic blacks $(62.2 \%$ (46 of 74); $p=0.004$ ) and nonHispanic whites (67.2\% (180 of 268); $p<0.001)[9,19]$. A longitudinal study (4-12 year follow up) conducted in a predominantly white cohort showed that $50 \%$ of participants with T1D seroconverted from ZnT8 AAb positive to negative status after a disease duration of 6.2 years [21]. Thus, as the median duration seen in our study is 7 years, it is likely that the $17.5 \% \mathrm{ZnT} 8 \mathrm{AAb}$ prevalence is an underestimation, and this is confirmed by the higher prevalence of ZnT8 AAbs (27.3\%) observed in participants with a disease duration of $<1$ year. These studies suggest that the frequency of the $\mathrm{ZnT} 8$ $\mathrm{AAb}$ is lower in indigenous African populations.

Previous studies involving both cross-sectional and longitudinal analyses $[8,21,22]$ have shown that ZnT8 $\mathrm{AAb}$ titres decline with increasing duration of disease. In the current study, we did not find a difference in ZnT8 $\mathrm{AAb}$ titres when comparing individuals with varying disease duration, but this may be a reflection of our small sample size and/or due to the type of assay used i.e. ELISA or radiobinding assays, as their sensitivity and specificity are known to differ [23].

The prevalence of GAD65 AAb positivity in this study was $51.3 \%$, with a median disease duration of 7 years. This frequency is similar to two other South African studies performed in black participants i.e. $44 \%$ [24] and $60 \%$ [25], in both of which disease duration was less than that of the current study - mean of 3.4 years and median of 5 years, respectively. However, it is lower than the GAD65 AAb frequencies seen in newly diagnosed White (European and American; 72-76\%) [26, 27], Japanese (60-70\%) [28], and African American (56-76\%) $[27,29]$ populations with T1D. Similarly, the prevalence of GAD65 AAbs was higher for South African white participants (median disease duration of 8.5 years) at $66 \%$ [25]. In addition, the GAD65 AAb prevalence seen in our study is higher than that seen in other sub-Saharan countries, namely Cameroon (34\%, disease duration: $<5$

Table 5 Logistic regression model for the determinants of ZnT8 AAb positivity

\begin{tabular}{llll}
\hline Dependent variable & Independent variable & Odds ratio (95\% Cl) & \\
\hline ZnT8 AAb positivity & Disease duration & $0.90(0.81-1.00)$ & 0.042 \\
& GAD65 AAb ${ }^{a}$ & $3.37(1.10-10.3)$ & 0.033 \\
& IA2 AAb & $8.63(2.82-26.4)$ & $<0.001$
\end{tabular}

For full model $p<0.001(\mathrm{n}=155),{ }^{\mathrm{a}} \mathrm{GAD} 65$ positive $=1$, GAD65 negative $=0 ;{ }^{\mathrm{b}} \mathrm{IA} 2$ positive $=1$, IA2 negative $=0$ 
Table 6 Multiple regression model for the determinants of age at diagnosis

\begin{tabular}{llll}
\hline Dependent variable & Independent variable & B value & $\boldsymbol{p}$ value \\
\hline Age at diagnosis & IA2 AAb positivity ${ }^{{ }^{a}}$ & -5.646 & 0.005 \\
& Gender $^{\mathrm{b}}$ & 2.639 & 0.052 \\
\hline
\end{tabular}

${ }^{a} I A 2$ AAb code $0=$ negative $a 1=$ positive ${ }^{b}$ Gender code $0=$ female and $1=$ male; for full model $p<0.005(\mathrm{n}=154) ; \mathrm{R}^{2}=0.056$

years) [30] and Tanzania (30\%, median disease duration of 3 years) [31].

Similarly, in our cohort we found the prevalence of IA2 AAbs (12.8\%) to be lower than the frequencies seen in newly diagnosed white (European: $58 \%$ and American: $63 \%$ ) [26, 27], Japanese (60-65\%) [28], and African American (42\%) [27] populations with T1D. In addition, higher IA2 AAb frequencies (41\%) were seen in a white South African population (median disease duration 8.5 years) [25]. However, the IA2 AAb frequencies noted in our study were similar to that reported in black South Africans (19\%, median disease duration of 5 years) [25], Tunisians (21\%, median disease duration of 3 years) [31] and Cameroonians (6.4 and $10 \%$; median disease durations $<5$ years and 23 days, respectively) $[30,32]$.

It is clear that the frequencies of AAbs vary greatly in different populations but tend to be lower in black African populations. The exact mechanism through which this occurs is unknown, however, Padoa et al. [24] hypothesised that the autoimmune response in black participants with T1D may be attenuated compared to other populations, thus resulting in a decreased frequency of AAbs and an older age at diagnosis in the black population.

The presence of ZnT8 AAbs has been associated with a younger age at diagnosis in European, American and Chinese studies $[4,8,12,17]$. In contrast, a British study found ZnT8 positivity to be associated with an older age at diagnosis while no association was seen in a study on newly diagnosed Czech children [14]. In our cohort, the association of ZnT8 AAb positivity with an earlier age at diagnosis was lost in multiple regression analysis with only IA2 remaining associated with an earlier age at diagnosis. The lack of association of $\mathrm{ZnT} 8 \mathrm{AAb}$ positivity with age at diagnosis is consistent with a study in newly diagnosed Czech children [25] and with the hypothesis that these AAbs are not disease-causing but

Table 7 Multiple regression model for the determinants of age at diagnosis

\begin{tabular}{llll}
\hline Dependent variable & Independent variable & B value & $\boldsymbol{p}$ value \\
\hline Age at diagnosis & $>1 \mathrm{AAb}^{\mathrm{a}}$ & -5.270 & 0.002 \\
& Gender $^{\mathrm{b}}$ & 2.539 & 0.059 \\
\hline
\end{tabular}

${ }^{a}>1$ AAb code: $0=<2$ AAbs present $1=>2$ AAbs, ${ }^{b}$ Gender code: $0=$ female and 1 $=$ male for full model $p<0.002(\mathrm{n}=154) ; \mathrm{R}^{2}=0.067$ rather reflect progression of the autoimmune response [26]. IA2 AAb positivity is associated with a more rapid progression to disease and are more commonly found in younger vs. older participants [17, 27]. However, in contrast to our study, Steck and colleagues [28] found that the presence of IA2 was not associated with age at diagnosis.

Individuals with two or three AAbs were found to have an earlier age at diagnosis than participants with either one or no AAbs. This is in agreement with a study which found that increasing number of AAbs in Italian adult participants with autoimmune diabetes were associated with a younger age at diagnosis [29]. This is likely due to an increased immune response resulting in a more rapid destruction of pancreatic $\beta$-cells and thus a decreased time to T1D onset.

Increasing duration of T1D has been shown to be associated with declining levels of ZnT8 AAbs [9, 14, 16, 30]. It is possible that the decline in AAb positivity over time is due to the continuous destruction of pancreatic $\beta$-cells. As the $\beta$-cells are destroyed, fewer antigens are presented to B cells and hence fewer AAbs are produced [31].

The limitations of this study were a relatively small sample size, lack of GAD65 and IA-2 AAb data for the entire cohort and a small proportion (12.2\%) of newly diagnosed ( $<1$ year) cases. In addition, this was a crosssectional study and therefore causation could not be tested and HLA genotyping was not performed in this study. In previous investigations ZnT8 AAb positivity was not associated with the HLA DR4/DQ8 haplotype in a Finnish population [11] and in British participants from the Type 1 Diabetes Genetics Consortium (T1DGC) [14]. However, a larger study on participants from the T1DGC (Asia-Pacific, European, North American, and Britain) found an association with the HLA DRB1*04:04 allele but not with the DRB1*04:01 allele [9], an association that was not observed in the earlier study restricted to the British T1DGC participants [14]. The frequency of DR alleles varies amongst different ethnic groups [32]. Thus, future studies are necessary to determine if the low $\mathrm{ZnT}$ 8 AAb prevalence seen in our study is due to variations in HLA DR allele frequencies in the South African black population compared to those seen in other populations.

\section{Conclusions}

In conclusion, ZnT8 AAb prevalence is significantly lower in black South Africans with T1D than in European and African American cohorts, but similar to that in other sub-Saharan African populations, suggesting that the autoimmune destruction process may be similar in different populations but the severity of the response may be weaker in the South African black population. Futhermore, the low ZnT8 AAb prevalence in our 
cohort suggests that this AAb may not be useful in identifying individuals at risk for T1D in this population, but this requires conformation in longitudinal studies.

\section{Acknowledgements}

We would like to acknowledge Prof Raal for allowing us access to the diabetic clinic, Sr Angeline Naidoo for blood draws and the participants for agreeing to partake in this study.

\section{Authors' contributions}

CJP designed the study. SBhola recruited participants, collected the data and prepared the first draft of the manuscript. CJP and EMC performed statistical analysis, writing and critical review of the manuscript. NJC was involved in critical review of the manuscript. SB provided access to participant samples and reviewed the manuscript. All authors read and approved the final manuscript.

\section{Funding}

This work was supported by a National Health Laboratory Service Research Trust Development Grant under Grant number 94398 and a University of the Witwatersrand Faculty of Health Sciences Faculty Research Committee Grant.

\section{Availability of data and materials}

The datasets used and/or analysed during the current study are available from the corresponding author on reasonable request.

\section{Declarations}

\section{Ethics approval and consent to participate}

The study protocol was approved by the University of the Witwatersrand Human Research Ethics Committee (clearance certificate numbers M180334 and M150885) and by the South African Blood Transfusion Services Research Ethics Committee (clearance certificate number 2014/19). Informed consent was obtained from all participants. The study protocol conformed to the Declaration of Helsinki ethical guidelines.

\section{Consent for publication}

Not applicable.

\section{Competing interests}

The authors declare no conflict of interest.

\section{Author details}

'Department of Chemical Pathology, Faculty of Health Sciences, University of the Witwatersrand, Johannesburg, South Africa. ${ }^{2}$ National Health Laboratory Service, Johannesburg, South Africa. ${ }^{3}$ Department of Medicine, Chris Hani Baragwanath Hospital, Faculty of Health Sciences, University of the Witwatersrand, Johannesburg, South Africa.

Received: 11 March 2021 Accepted: 22 June 2021

Published online: 16 July 2021

\section{References}

1. Foulis AK, Liddle CN, Farquharson MA, Richmond JA, Weir RS. The histopathology of the pancreas in type 1 (insulin-dependent) diabetes mellitus: a 25-year review of deaths in patients under 20 years of age in the United Kingdom. Diabetologia. 1986;29(5):267-74.

2. Simmons KM, Michels AW. Type 1 diabetes: A predictable disease. World J Diabetes. 2015;6(3):380-90.

3. Tiberti C, Yu L, Lucantoni F, Panimolle F, Spagnuolo I, Lenzi A, et al. Detection of four diabetes specific autoantibodies in a single radioimmunoassay: an innovative high-throughput approach for autoimmune diabetes screening. Clin Exp Immunol. 2011;166(3):317-24.

4. Wenzlau JM, Juhl K, Yu L, Moua O, Sarkar SA, Gottlieb P, et al. The cation efflux transporter ZnT8 (SIc30A8) is a major autoantigen in human type 1 diabetes. Proc Natl Acad Sci U S A. 2007;104(43):17040-5.

5. Seve $M$, Chimienti F, Devergnas S, Favier A. In silico identification and expression of SLC30 family genes: an expressed sequence tag data mining strategy for the characterization of zinc transporters' tissue expression. BMC Genomics. 2004;5(1):32.

6. Carroll RJ, Hammer RE, Chan SJ, Swift HH, Rubenstein AH, Steiner DF. A mutant human proinsulin is secreted from islets of Langerhans in increased amounts via an unregulated pathway. Proc Natl Acad Sci U S A. 1988;85(23): 8943-7.

7. Mitchell RK, Hu M, Chabosseau PL, Cane MC, Meur G, Bellomo EA, et al. Molecular Genetic Regulation of Slc30a8/ZnT8 Reveals a Positive Association With Glucose Tolerance. Mol Endocrinol. 2016;30(1):77-91.

8. Vaziri-Sani F, Oak S, Radtke J, Lernmark K, Lynch K, Agardh CD, et al. ZnT8 autoantibody titers in type 1 diabetes patients decline rapidly after clinical onset. Autoimmunity. 2010;43(8):598-606

9. Wenzlau JM, Frisch LM, Hutton JC, Fain PR, Davidson HW. Changes in Zinc Transporter 8 Autoantibodies Following Type 1 Diabetes Onset: The Type 1 Diabetes Genetics Consortium Autoantibody Workshop. Diabetes Care. 2015;38 Suppl 2:S14-20.

10. Fabris M, Zago S, Liguori M, Trevisan MT, Zanatta M, Comici A, et al. Antizinc transporter protein 8 autoantibodies significantly improve the diagnostic approach to type 1 diabetes: an Italian multicentre study on paediatric patients. Auto Immun Highlights. 2015;6(1-2):17-22.

11. Salonen KM, Ryhanen S, Harkonen T, Ilonen J, Knip M, Finnish Pediatric Diabetes R. Autoantibodies against zinc transporter 8 are related to age, metabolic state and HLA DR genotype in children with newly diagnosed type 1 diabetes. Diabetes Metab Res Rev. 2013;29(8):646-54.

12. Yang L, Luo S, Huang G, Peng J, Li X, Yan X, et al. The diagnostic value of zinc transporter 8 autoantibody (ZnT8A) for type 1 diabetes in Chinese. Diabetes Metab Res Rev. 2010;26(7):579-84.

13. Kawasaki E, Uga M, Nakamura K, Kuriya G, Satoh T, Fujishima K, et al. Association between anti-ZnT8 autoantibody specificities and SLC30A8 Arg325Trp variant in Japanese patients with type 1 diabetes. Diabetologia. 2008;51(12):2299-302.

14. Howson JM, Krause S, Stevens H, Smyth DJ, Wenzlau JM, Bonifacio E, et al. Genetic association of zinc transporter 8 (ZnT8) autoantibodies in type 1 diabetes cases. Diabetologia. 2012;55(7):1978-84.

15. Gomes KF, Semzezem C, Batista R, Fukui RT, Santos AS, Correia MR, et al. Importance of Zinc Transporter 8 Autoantibody in the Diagnosis of Type 1 Diabetes in Latin Americans. Sci Rep. 2017;7(1):207.

16. Kawasaki E, Oikawa Y, Okada A, Kanatsuna N, Kawamura T, Kikuchi T, et al. Zinc transporter 8 autoantibodies complement glutamic acid decarboxylase and insulinoma-associated antigen-2 autoantibodies in the identification and characterization of Japanese type 1 diabetes. J Diabetes Investig. 2020; 11(5):1181-87.

17. Niechcial E, Rogowicz-Frontczak A, Pilacinski S, Fichna M, Skowronska B, Fichna $\mathrm{P}$, et al. Autoantibodies against zinc transporter 8 are related to age and metabolic state in patients with newly diagnosed autoimmune diabetes. Acta Diabetol. 2018;55(3):287-94.

18. Bahendeka S, Wesonga R, Were TP, Nayangabyaki C. Autoantibodies and HLA class II DR-DQ genotypes in Ugandan children and adolescents with type 1 diabetes mellitus. Int J Diabetes Dev Ctries. 2019;39:39-46.

19. Balcha SA, Demisse AG, Mishra R, Vartak T, Cousminer DL, Hodge KM, et al. Type 1 diabetes in Africa: an immunogenetic study in the Amhara of NorthWest Ethiopia. Diabetologia. 2020;63(10):2158-68.

20. Sunni M, Noble JA, Yu L, Mahamed Z, Lane JA, Dhunkal AM, et al. Predominance of DR3 in Somali children with type 1 diabetes in the twin cities, Minnesota. Pediatr Diabetes. 2017;18(2):136-42.

21. Wenzlau JM, Walter M, Gardner TJ, Frisch LM, Yu L, Eisenbarth GS, et al. Kinetics of the post-onset decline in zinc transporter 8 autoantibodies in type 1 diabetic human subjects. J Clin Endocrinol Metab. 2010;95(10):4712-9.

22. Kawasaki E, Oikawa Y, Okada A, Kanatsuna N, Kawamura T, Kikuchi T, et al. Different interaction of onset age and duration of type 1 diabetes on the dynamics of autoantibodies to insulinoma-associated antigen-2 and zinc transporter 8. J Diabetes Investig. 2021;12(4):510-5.

23. Kawasaki E, Eisenbarth GS. High-throughput radioassays for autoantibodies to recombinant autoantigens. Front Biosci. 2000;5:E181-90.

24. Padoa CJ, Rheeder P, Pirie FJ, Motala AA, van Dyk JC, Crowther NJ. Identification of a subgroup of black South Africans with type 1 diabetes who are older at diagnosis but have lower levels of glutamic acid decarboxylase and islet antigen 2 autoantibodies. Diabet Med. 2020;37(12):2067-74.

25. Petruzelkova L, Ananieva-Jordanova R, Vcelakova J, Vesely Z, Stechova K, Lebl J, et al. The dynamic changes of zinc transporter 8 autoantibodies in Czech children from the onset of Type 1 diabetes mellitus. Diabet Med. 2014;31(2):165-71. 
26. Williams $\mathrm{CL}$, Long $\mathrm{AE}$. What has zinc transporter 8 autoimmunity taught us about type 1 diabetes? Diabetologia. 2019;62(11):1969-76.

27. Kohler M, Beyerlein A, Vehik K, Greven S, Umlauf N, Lernmark A, et al. Joint modeling of longitudinal autoantibody patterns and progression to type 1 diabetes: results from the TEDDY study. Acta Diabetol. 2017;54(11):1009-17.

28. Steck AK, Johnson K, Barriga KJ, Miao D, Yu L, Hutton JC, et al. Age of islet autoantibody appearance and mean levels of insulin, but not GAD or IA-2 autoantibodies, predict age of diagnosis of type 1 diabetes: diabetes autoimmunity study in the young. Diabetes Care. 2011;34(6):1397-9.

29. Lampasona V, Petrone A, Tiberti C, Capizzi M, Spoletini M, di Pietro S, et al. Zinc transporter 8 antibodies complement GAD and IA-2 antibodies in the identification and characterization of adult-onset autoimmune diabetes: Non Insulin Requiring Autoimmune Diabetes (NIRAD) 4. Diabetes Care. 2010;33(1):104-8.

30. Kawasaki E, Nakamura K, Kuriya G, Satoh T, Kobayashi M, Kuwahara H, et al. Zinc transporter 8 autoantibodies in fulminant, acute-onset, and slow-onset patients with type 1 diabetes. Diabetes Metab Res Rev. 2011;27(8):895-8.

31. Cheng BW, Lo FS, Wang AM, Hung CM, Huang CY, Ting WH, et al. Autoantibodies against islet cell antigens in children with type 1 diabetes mellitus. Oncotarget. 2018;9(23):16275-83.

32. Lombard Z, Brune AE, Hoal EG, Babb C, Van Helden PD, Epplen JT, et al. HLA class II disease associations in southern Africa. Tissue Antigens. 2006; 67(2):97-110.

\section{Publisher's Note}

Springer Nature remains neutral with regard to jurisdictional claims in published maps and institutional affiliations.

Ready to submit your research? Choose BMC and benefit from:

- fast, convenient online submission

- thorough peer review by experienced researchers in your field

- rapid publication on acceptance

- support for research data, including large and complex data types

- gold Open Access which fosters wider collaboration and increased citations

- maximum visibility for your research: over $100 \mathrm{M}$ website views per year

At $\mathrm{BMC}$, research is always in progress.

Learn more biomedcentral.com/submissions 